\title{
La relevancia de los enfoques de género en las Relaciones Internacionales y su aplicación al estudio de las mujeres en Medio Oriente
}

\author{
Alejandra Gutiérrez Luna alegutierrezluna@politicas.unam.mx. \\ Universidad Nacional Autónoma de México (México)
}

Recibido: $29 / 07 / 2020$

Aceptado: $28 / 10 / 2021$

Resumen: En las últimas tres décadas, el estudio de la influencia del papel de las mujeres en los sucesos internacionales ha ido en aumento. Es por ello que el objetivo de este escrito es reflexionar sobre la relevancia y aplicación de los enfoques de género en los estudios internacionales. Se argumenta que las corrientes de géneroson relevantes e incluso indispensables en las Relaciones Internacionales, debido a que cuestionan la construcción del conocimiento y generan investigaciones que promueven la transformación social en términos de equidad de género y participación política, como se muestra con los casos de los activismos feministas en Palestina y Egipto.

Palabras clave: feminismo, género, Medio Oriente, mujeres, actores no estatales.

* Cómo citar: Gutiérrez Luna, A. (2021). La relevancia de los enfoques de género en las Relaciones Internacionales y su aplicación al estudio de las mujeres en Medio Oriente. Relaciones Internacionales, 30 (61), 137.

https://doi.org/10.24215/23142766e137

Editor: Juan Alberto Rial, Instituto de Relaciones Internacionales Facultad de Ciencias Jurídicas y Sociales (Universidad Nacional de La Plata)
Entidad editora: Relaciones

Internacionales, es una publicación del Instituto de Relaciones Internacionales (Facultad de Ciencias Jurídicas y Sociales (Universidad Nacional de La Plata Argentina) Vol30- no 61/2021

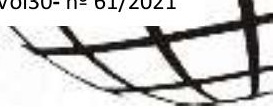




\title{
The relevance of gender approaches in International Rela- tions and their application to the study of women in the Middle East
}

\section{Alejandra Gutiérrez Luna ${ }^{1}$}

\begin{abstract}
In the last three decades, the study of the influence that the role of women has had on international events has increased.Therefore, the aim of this text is to reflect on the relevance and application of gender approaches in international studies. It is argued that gender approaches are relevant and even key in International Relations, because they question the construction of knowledge and are the source of research that promotes social transformation in terms of gender equality and political participation, as shown by the cases of feminist activisms in Palestine and Egypt.
\end{abstract}

Key words: feminism, gender, Middle East, women, non-state actors.

1 Doctora en Ciencias Políticas y Sociales con orientación en Relaciones Internacionales por la Universidad Nacional Autónoma de México. Profesora en la Facultad de Estudios Superiores Acatlán y en la Facultad de Ciencias Políticas y Sociales de la UNAM. 


\section{Introducción}

Desde su surgimiento, al finalizar la Primera Guerra Mundial ${ }^{2}$, hasta las décadas de los años sesenta y setenta, la disciplina de las Relaciones Internacionales (RR.II.) se enfocó en el análisis del Estado como su principal y prácticamente única figura; sin embargo, algunos teóricos, como Robert O. Keohane y Joseph S. Nye (1971), han destacado -por los cuestionamientos que plantearon- dicho carácter estatocéntrico, de modo que se abrió paso al estudio de la influencia de actores no estatales, como los movimientos sociales, las organizaciones no gubernamentales, los individuos, las mujeres y las empresas transnacionales, entre otros, en los sucesos internacionales.

Este artículo hace énfasis en el análisis de las mujeres como actores internacionales y tiene como objetivo reflexionar sobre la relevancia y aplicación de los enfoques de género en los estudios internacionales. Se sostiene que los enfoquesde géneroson pertinentes e incluso indispensables en el estudio de las RR.II. debido a que cuestionan la construcción del conocimiento y generan investigaciones que promueven la transformación social en términos de equidad de género y participación política. Asimismo, ofrecen una visión crítica de la realidad y las relaciones sociales en los niveles local y transnacional. El método empleado es el estudio de los casos de los activismos feministas en Palestina y Egipto. La selección responde a que la autora ha realizado investigaciones previas y trabajo de campo en estos dos Estados.

El texto está organizado en tres partes. En la primera se realiza unareflexión breve en torno a la relevancia y aplicación de los enfoques de género en el estudio de las Relaciones Internacionales; posteriormente, se vincula a las corrientes de género con el activismo de las mujeres palestinas en el marco de la ocupación israelí; $y$, en la tercera parte, se aborda el caso del feminismo egipcio a una década de la llamada Primavera Árabe, desde una perspectiva internacional.

\section{Los enfoques de género en Relaciones Internacionales}

Los estudios de género analizan la forma en que los fenómenos sociopolíticos afectan de manera diferenciada a hombres y mujeres. También estudian la condición de las mujeres y las vías para lograr su emancipación. El feminismo y el género comparten temas de estudio; sin embargo, tienen algunas diferencias. Las principales tienen que ver con el alto contenido político y activista del feminismo, así como el carácter académico del género.

Los estudios de género en Relaciones Internacionales aparecieron a finales de los años ochenta en el contexto de grandes transformaciones en el sistema internacional con el objetivo de analizar la manera en que los sujetos sociales se ven influidos de manera diferenciada por las guerras, las luchas nacionalistas y demás sucesos. Las corrientes de género y

\footnotetext{
2 El estudio académico de las Relaciones Internacionales era embrionario antes de este gran acontecimiento. En la segunda mitad del siglo XIX, las RR.II. seguían sin ser identificadas como un foco de estudio autónomo; lo que ahora se entiende como RR.II. era visto simplemente como una faceta o extensión de disciplinas, como la Ciencia Política y la Sociología (Ochoa, et al. 2016: 33) .
} 
feminismo en Relaciones Internacionales han analizado la intervención de algunas mujeres en conflictos, luchas nacionalistas, revueltas populares, así como la influencia de estos últimos en la conformación de las estructuras de género.

Los enfoquesde género replantean el papel de las mujeres como actores internacionales, al tiempo que las causas feministas se convierten en estandarte de diversos movimientos sociales. Los actores de la sociedad civil global ${ }^{3}$, como los movimientos feministas, han adquirido poder, agencia y capacidad para incidir en procesos de cambio político ante la incapacidad de los sistemas políticos nacionales para controlar diversos problemas. La sociedad civil global se ha convertido en la expresión organizada de los valores e intereses de la sociedad (Castells, 2008: 78).

Si bien la literaturade género no es una escuela de pensamiento en las Relaciones Internacionales, forma parte de las recientes tendencias de estudio y análisis cuya importancia y aplicación ha ido en aumento.Su aparición ocurrió en 1988 en la Conferencia Millenium: Journal of International Studies (Milenio: Revista de Estudios Internacionales) en The London School of Economics. A partir de ello, diversas teóricas -como Jean Elshtain, con aportaciones valiosas sobre el papel de las mujeres en las guerras; Cinthya Enloe, reconocida internacionalmente por su trabajo acerca del género y el militarismo, sobre todo por su libro Bananas, Beaches and Bases: Making Feminist Sense of International Politics (2014); Christine Sylvester, quien ha examinado la historia de los esfuerzos feministas para incluir las relaciones de género en el estudio de las relaciones internacionales; Sandra Withworth, por sus artículos y capítulos de libros sobre temas como el género en la política exterior canadiense y los derechos humanos; Ann Tickner, quien ha propuesto una reinterpretación teórica de las Relaciones Internacionales desde una perspectiva feminista; y Jacquie True, por sus investigaciones sobre mujeres, paz y seguridad, economía política de la violencia contra las mujeres y violencia sexual en los conflictos de Asia Pacífico- comenzaron a incorporar nuevos temas al estudio de las Relaciones Internacionales, reanalizando términos clave como "poder" y "soberanía", y cuestionando las construcciones masculinas de esos conceptos desde distintas corrientes.

Las investigaciones de género y feminismo en las Relaciones Internacionales han sido construidas en torno a vacíos teóricos que existen principalmente, debido a que las tendencias clásicas de las Relaciones Internacionales estudian temas como las causas de las guerras, la expansión del comercio, las negociaciones entre gobiernos o la cooperación internacional, pero pocas veces hacen referencia a los individuos y a su influencia en tales procesos. De igual forma, algunas corrientes ignoran las construcciones sociales de lo femenino y lo masculino, aun cuando éstas se encuentran de forma implícita en la mayor parte de los acontecimientos globales.

Pese a esto, diversas tendencias feministas se han aplicado al estudio de temas concernientes a las Relaciones Internacionales, como el papel de las mujeres en los conflictos

3 Martin Shaw (1994: 648) define a la sociedad civil como una red de instituciones donde la sociedad se representa a sí misma frente al Estado, de manera local e internacional. Así, la sociedad civil se define por la relación que tiene con el Estado. 
armados y en las revueltas populares, y su impacto en las relaciones de género. Cada una de estas tendencias ofrece interpretaciones acerca de los factores de opresión que obstaculizan la visibilización de las mujeres en procesos internacionales. En el cuadro 1 se sintetizan estas perspectivas.

Cuadro 1. Corrientes de género aplicadas al estudio de las Relaciones Internacionales

\begin{tabular}{|c|c|c|c|c|}
\hline Corriente & Temas de estudio & Exponentes & $\begin{array}{l}\text { Conceptos } \\
\text { centrales }\end{array}$ & Similitudes \\
\hline Liberal & $\begin{array}{l}\text { Refugiados, } \\
\text { desigualdad en los } \\
\text { ingresos, impacto de } \\
\text { la presencia de } \\
\text { mujeres en lo militar. }\end{array}$ & $\begin{array}{l}\text { Betty Friedan, } \\
\text { Jane Richards }\end{array}$ & $\begin{array}{l}\text { Libertad, } \\
\text { igualdad }\end{array}$ & \\
\hline Crítica & $\begin{array}{l}\text { Razas, clases, } \\
\text { sexualidad, etnicidad, } \\
\text { religión. }\end{array}$ & Sandra Whitworth & Ideas & $\begin{array}{l}\text { Brindan } \\
\text { importancia } \\
\text { a las ideas }\end{array}$ \\
\hline $\begin{array}{l}\text { Constructivis } \\
\text { ta }\end{array}$ & $\begin{array}{l}\text { Importancia de las } \\
\text { ideas, trabajo en el } \\
\text { hogar. }\end{array}$ & Elisabeth Prugl & & género. \\
\hline Posmoderna & $\begin{array}{l}\text { Relación entre } \\
\text { conocimiento y } \\
\text { poder. } \\
\text { Deconstrucción del } \\
\text { concepto 'mujer'. }\end{array}$ & $\begin{array}{l}\text { Marysia Zalewski, } \\
\text { Jean Elshtain, } \\
\text { Christine Syl- } \\
\text { vester, Judith But- } \\
\text { ler }\end{array}$ & \multirow[t]{2}{*}{$\begin{array}{l}\text { Lenguaje, } \\
\text { conocimiento, } \\
\text { realismo, } \\
\text { ciencia, } \\
\text { experiencias }\end{array}$} & \multirow{2}{*}{$\begin{array}{l}\text { Critican que } \\
\text { el } \\
\text { conocimient } \\
\text { o sea } \\
\text { construido } \\
\text { en torno a lo } \\
\text { masculino y } \\
\text { por los } \\
\text { poderosos. }\end{array}$} \\
\hline $\begin{array}{l}\text { Punto de } \\
\text { vista }\end{array}$ & $\begin{array}{l}\text { Crítica al realismo, } \\
\text { construcción del } \\
\text { conocimiento. }\end{array}$ & $\begin{array}{l}\text { Christine Syl- } \\
\text { vester, Ann Tick- } \\
\text { ner, Cynthia Enloe }\end{array}$ & & \\
\hline
\end{tabular}




\begin{tabular}{|c|c|c|c|c|}
\hline Empírica & $\begin{array}{l}\text { Mujeres en la ciencia, } \\
\text { experiencias de } \\
\text { mujeres en relaciones } \\
\text { internacionales, } \\
\text { importancia de la } \\
\text { educación. }\end{array}$ & $\begin{array}{l}\text { Sandra Harding, } \\
\text { Cynthia Enloe }\end{array}$ & & \\
\hline Poscolonial & $\begin{array}{l}\text { Relaciones coloniales } \\
\text { de dominación. }\end{array}$ & Chandra Mohanty & \multirow{2}{*}{$\begin{array}{l}\text { Imperialismo, } \\
\text { colonialismo, } \\
\text { estereotipos, } \\
\text { capitalismo }\end{array}$} & \multirow{2}{*}{$\begin{array}{l}\text { Las } \\
\text { estructuras, } \\
\text { como el } \\
\text { capitalismo y } \\
\text { el } \\
\text { imperialismo } \\
\text {, contribuyen } \\
\text { a la opresión } \\
\text { de algunas } \\
\text { mujeres. }\end{array}$} \\
\hline $\begin{array}{l}\text { Socialista/ } \\
\text { Marxista }\end{array}$ & $\begin{array}{l}\text { Capitalismo, } \\
\text { estructuras de } \\
\text { producción, } \\
\text { desigualdad } \\
\text { socioeconómica. }\end{array}$ & $\begin{array}{l}\text { Heidi Hartmann, } \\
\text { Alison Jaggar }\end{array}$ & & \\
\hline Radical & $\begin{array}{l}\text { Nociones de la } \\
\text { diferencia, paz y } \\
\text { cooperación. }\end{array}$ & $\begin{array}{l}\text { Shulamit Fire- } \\
\text { stone, Eva Figes, } \\
\text { Kate Millet }\end{array}$ & $\begin{array}{l}\text { Patriarcado, } \\
\text { diferencias }\end{array}$ & \\
\hline
\end{tabular}

Fuente: Elaboración propia con base en Salomón, 2002; Tickner, 2008; True, 2008 y Villarroel, 2007

Como se puede observar en el cuadro, el feminismo liberal sugiere que las mujeres han estado subrepresentadas y excluidas de la esfera pública, de manera que su inclusión en los asuntos internacionales disminuiría las desigualdades de género. Este tipo de feminismo es el que se conoce como el de la igualdad, ya que parte de la idea de que las mujeres y los hombres tienen las mismas capacidades. Defiende los valores de libertad e igualdad de oportunidades y argumenta que las mujeres son oprimidas porque no son tratadas igual que los hombres (Villarroel, 2007: 68).

La corriente crítica considera que el género es una construcción social que ha reproducido las desigualdades entre hombres y mujeres. En general, esta tendencia brinda importancia al contexto y a los procesos históricos en los que se desarrolla (D'Amico, 1994: 63). Considera que las concepciones de género influyen en las políticas de organismos internacionales.

El enfoqueconstructivista se centra en el estudio de los procesos mediante los cuales las ideas acerca del género influyen en la política internacional, así como la forma en la que estos establecen ideas acerca de las relaciones de género (Tickner, 2008: 26). En lo que toca alposmodernismo, éste pretende deconstruir el concepto "mujer" con el objetivo de explorar y desarrollar sus significados (Whitworth, 1994: 81), ya que considera que la situación 
de las mujeres es muy diversa. Supone que la realidad es entendida mediante el uso del lenguaje, que el conocimiento se ha basado en la forma de vida pública de los hombres (Tickner, 2008: 26) y no cree en los procesos universales (definiciones, puntos de vista, situaciones).

El feminismo de punto de vista (feminsit standpoint) argumenta que la construcción del conocimiento, a partir de las experiencias de las mujeres, brinda una imagen más integral del mundo, al mismo tiempo sostiene que la ciencia refleja la posición dominante de los hombres en la vida social (Villarroel, 2007: 70). Además, realiza una reinterpretación teórico-práctica de las relaciones internacionales a través de una mirada feminista (Salomón, 2002: 35) y critica al realismo político, debido a que legitima el orden impuesto. Específicamente, Ann Tickner (1988: 431) propone examinar los seis puntos del realismo político de Hans Morgenthau (1997), debido a que hacen una descripción parcial de la política internacional al privilegiar las características masculinas. Las dicotomías occidentales como objetividad vs subjetividad, razón vs emoción, mente vs cuerpo, cultura vs naturaleza, público vs privado, entre otras, han sido utilizadas para estereotipar y establecer diferencias entre lo masculino y lo femenino.

Tickner (1988: 437) propone reformular, por ejemplo, el principio número dos de Morgenthau 4 por uno que posea una perspectiva feminista: el interés nacional es multidimensional, por lo que no puede ser definido solamente en términos de poder. En el mundo contemporáneo el interés nacional requiere soluciones cooperativas más que de suma cero.

Por otra parte, la corriente empírica considera que la ciencia ha desempeñado una división sexual y que las experiencias de las mujeres han sido excluidas de procesos internacionales, como son las guerras y los conflictos. Afirma que el androcentrismo ${ }^{5}$ en la investigación científica es un sesgo que puede erradicarse con una aproximación adecuada a los métodos científicos (Harding, 1986: 36).

El feminismo radicalsostiene que las relaciones de dominación de los hombres hacia las mujeres y las estructuras patriarcales constituyen una de las formas de opresión más relevantes. Diversos teóricos de la escuela radical estudian temas de guerra y paz, procesos en los cuales hombres y mujeres tienen funciones totalmente distintas (Whitworth, 1994: 78); incluso algunos autores consideran que las mujeres son más pasivas y los hombres más violentos. Este es el llamado feminismo de la diferencia. No persigue la igualdad, sino enfatizar los valores femeninos.

La tendencia socialista/marxista afirma que las injusticias en contra de las mujeres han

\footnotetext{
4 El principio número dos de Morgenthau (1997: 5) dicta que: el rasgo principal del realismo político es el concepto de interés, definido en términos de poder que infunde un orden racional al objeto de la política y de ese modo hace posible la comprensión teórica de la política. El realismo político hace hincapié en lo racional, lo objetivo y lo no emocional.

$5 \mathrm{El}$ androcentrismo consiste en la asimilación de la realidad en términos masculinos. De manera específica, las investigaciones androcéntricas interpretan los fenómenos sociopolíticos desde una óptica masculina e ignoran las experiencias de las mujeres. Se trata de estudios que posicionan a las mujeres en un nivel pasivo.
} 
surgido como consecuencia del orden económico actual (el capitalismo).Por último, el feminismo poscolonial o del Tercer Mundo sostiene que la opresión de las mujeres tiene su origen en el racismo y el imperialismo. Esta corriente considera que algunos medios de comunicación y las feministas occidentales construyen ideas erróneas y estereotipadas acerca de las mujeres del Tercer Mundo e ignoran la complejidad de su situación. En este sentido, las mujeres de Medio Oriente son vistas como víctimas incapaces de modificar sus propias realidades, lo que produce que esa misma dicotomía que existe entre hombres y mujeressurja entre mujeres occidentales y mujeres del Tercer Mundo.

La mayor parte de las corrientes feministas en las Relaciones Internacionales comparten un carácter normativo (True, 2008: 408), pues pretenden analizar las relaciones de género con el fin de transformar las representaciones tradicionalesfemeninas y construir principios teóricos que guíen la práctica política. Al incorporar los estudios feministas en las Relaciones Internacionales, se favorece una interpretación de la realidad menos marcada por valores masculinos y se generan procesos de transformación social mediante los cuales es posible disminuir la exclusión de las mujeres.

Otras similitudes entre los enfoques mencionados tienen que ver con los temas de estudio. Las corrientes crítica y constructivista tratan asuntos relacionados con las ideas construidas socialmente; el feminismo posmoderno, el de punto de vista y el empíricoversan sobre el desempeño de las mujeres en la ciencia, las críticas al realismo político y la importancia del lenguaje y del conocimiento; por último, las tendencias de Tercer Mundo y marxista estudian la influencia del colonialismo, el imperialismo y el capitalismo en la opresión de las mujeres.

Las corrientes difieren en diversos aspectos. Por mencionar algunos, el feminismo liberal defiende los valores de la igualdad. Esto quiere decir que para que se erradique la opresión, las mujeres deben ser tratadas igual que los hombres; en cambio, el feminismo radicalopta por las nociones de la diferencia. Sostiene que las mujeres deberían ocupar puestos de poder, porque aportan una mirada más pacífica y cooperativa. El feminismo posmoderno hace una crítica a la corriente radical debido al esencialismo con el que esta última estudia las relaciones de género. Es decir, al hablar de mujer no se puede hacer referencia a una definición general que no tome en cuenta las diferencias que existen entre ellas, es por ello que, de acuerdo con el posmodernismo, es necesario deconstruir la categoría mujer (Whitworth, 1994: 81).

Por último, es pertinente rescatar que el estudio del género trata tanto de las categorías socialmente construidas de mujeres y feminidad, como las de hombres y masculinidad. Los estudios de género contemporáneos que incorporan un análisis interseccional, el cual sostiene que el género no puede entenderse de manera aislada de otras categorías de identidad y relaciones de desigualdad, reconocen que hay múltiples géneros debido a que raza, etnia, clase, sexualidad y otras características culturales dan forma a las identidades y representaciones de género (Runyan y Peterson, 2014: 3). 


\section{El activismo feminista palestino en el marco de la ocupación israelí}

Numerosos estudios han marginado el papel de las mujeres de las luchas nacionalistas, los conflictos internacionales e incluso de las revueltas populares; sin embargo, es necesario entender la manera en la que hombres y mujeres se ven afectados de manera diferenciada por estos procesos. Al hacer visibles las experiencias femeninas y masculinas, y la forma en que se distinguen, es posible integrar el tema del género en el estudio de diversos fenómenos globales.

Las cuestiones de género en Palestina son específicas y muy diferentes a las que se presentan en el resto de los países de Medio Oriente. Esto se debe principalmente a que la población vive en constante peligro como resultado de la ocupación israelí ${ }^{6}$, lo que ha impulsado un cambio radical en las funciones que tradicionalmente realizaban las mujeres.

En los primeros meses de 2020, el presidente estadounidense, Donald Trump, dio a conocer un plan con el cual Israel podría anexarseun $30 \%$ del territorio palestino (el Valle del Jordán, el Gran Jerusalén y los principales bloques de asentamientos donde viven 600 , 000 colonos israelíes), ello llevaría a que el eventual Estado palestino se reduciría al $15 \%$ de la Palestina del Mandato británico y consistiría en cuatro segmentos sin continuidad territorial y sin plena soberanía, ya que Israel controlaría sus fronteras y sus principales recursos (Álvarez-Ossorio, 2020).

Ante este panorama, el desempeño de las palestinas puede analizarse desde dos perspectivas: la primera, la causa nacionalista llevada a cabo mediante actos pacíficos y militares, y la segunda, a partir de la evolución de las relaciones de género en el contexto de la ocupación, lo que implica una transformación de los espacios destinados a las mujeres. Algunas de ellas han desafiado los prototipos de género al alejarse de las labores privadas y domésticas y participar en el espacio público; por ejemplo, en concentraciones, protestas, movimientos, proyectos, organizaciones no gubernamentales, campañas de alfabetización y acciones militares tradicionalmente masculinas; de manera que han logrado mantener su presencia en la lucha.

Sin embargo, Eileen Kuttab (2009: 106) considera que la lucha nacionalista femenina en Palestina es una condición necesaria, pero no suficiente, para su emancipación. De acuerdo con esta autora, la relación entre feminismo y nacionalismo en el contexto de la cuestión palestina ha sido malinterpretada debido a la visión feminista occidental que ignora la realidad colonial e imperial que viven estas mujeres. En otras palabras, el activismo femenino no es garantía para que surja un cambio en las relaciones de género que desfavorecen

\footnotetext{
6 Palestina vivió bajo el mandato de Gran Bretaña impuesto por la Sociedad de Naciones desde el fin de la Primera Guerra Mundial hasta 1947, año en que la Organización de las Naciones Unidas (ONU) aprobó el Plan para la Partición de Palestina, que establecía la creación, en territorio palestino, de un Estado árabe y uno judío, el cual se expandió más allá de lo que dictaba el plan. Esto tuvo como consecuencia el estallido de la primera guerra árabe-israelí en 1948, la creación del Estado de Israel y el desplazamiento de aproximadamente dos terceras partes de la población palestina, quienes se refugiaron principalmente en Siria, Líbano y Jordania. Posteriormente, se desataron otras guerras regionales como la de 1967, luego de la cual Israel ocupó el $78 \%$ de la tierra palestina.
} 
a las mujeres, aunque sí es un hecho que su participación es un incentivo para que ocurran cambios que eleven su estatus.

El feminismo es de crucial importancia para las redes y organizaciones que defienden los derechos de las palestinas; por ejemplo, de las prisioneras, ya que desde el feminismo se desestabilizan las instituciones que dependen de la reproducción de la desigualdad e injusticia y se critica las instituciones y prácticas que favorecen la violencia contra las mujeres que están expuestas al poder policial (en este caso al ejército israelí) por el simple hecho de manifestarse (Butler, 2014: 10).

De los enfoques de génerorevisados en el apartado anterior, el feminismo poscolonial o del Tercer mundo se ajusta al estudio de las mujeres palestinas debido al contexto social e histórico en que se han desarrollado. Esta tendencia considera al imperialismo y al colonialismo7 como dos de los procesos cruciales mediante los cuales se constituyen las identidades; asimismo, otorga gran importancia a la relación entre feminismo, antirracismo y luchas nacionalistas.

No todas las corrientes de género son adecuadas para analizar de manera integral la situación de las mujeres palestinas, ya que dicha situación supone una paradoja: las palestinas se encuentran apegadas al sistema patriarcal, al tiempo que muestran gran capacidad de resistencia ante la amenaza que representa la ocupación israelí. Es en particular el enfoque poscolonial o del Tercer mundo, según el cual la opresión de las mujeres tiene su origen en el racismo y el imperialismo, el que resulta útil para entender la realidad, debido a que permite atenuar la visión occidentalizada y estereotipada acerca de la vulnerabilidad y victimización de las mujeres de Medio Oriente, en general, y de las palestinas, en particular visión que en gran parte proviene del supuesto de que el islam es el único factor de opresión femenina.

El feminismo palestino se ha dividido en dos corrientes: la secular y la islamista. La islamista nació en el decenio de los años setenta y abarca aspectos sociales, económicos, políticos y religiosos. Esta corriente impulsa a las organizaciones a asesorar a las mujeres en tareas como bordar y trabajar como secretarias. Fomenta la incorporación de las mujeres al mercado de trabajo para contribuir a la economía familiar, siempre y cuando no les impida portar el velo al salir del hogar. Amal Zamili, quien ha coordinado organizaciones en la Franja de Gaza, ha sido una de las representantes de este feminismo. Por otro lado, el feminismo secular no ignora el componente religioso, pero no se basa en el islam tradicional. Esta tendencia nació en el contexto del mandato británico en Palestina y tuvo un auge en el decenio de los años setenta con el trabajo de algunas organizaciones. El feminismo secular, contrario

7 Chandra Mohanty (2008: 112) , una de las pioneras en el estudio de las mujeres del Tercer mundo, estudia al colonialismo no sólo como el dominio extranjero de un territorio, sino como una interpretación discursiva de colonización que hace referencia a 'una cierta forma de apropiación y codificación de "producción académica" y "conocimiento" acerca de las mujeres en el Tercer mundo por medio de categorías analíticas particulares. Estas categorías, empleadas en escritos específicos sobre el tema, toman como referencia los intereses feministas tal como han sido articulados en Estados Unidos y Europa Occidental'. La autora propone resaltar "los efectos similares de varias estrategias textuales utilizadas por escritoras que codifican al "Otro" como no occidental y, por tanto, (implícitamente) a sí mismas como [occidentales]'. 
al islamista, no define ni establece las labores de las mujeres en la familia, sino que pugna por una participación femenina más activa en la lucha nacionalista (Abu Ghaida, 1993: 18).

Respecto a la perspectiva de organismos internacionales, como la ONU, acerca de la presencia de las mujeres en procesos de paz, destaca la Resolución 1325 del Consejo de Seguridad del año 2000, la cual reconoce la labor de las mujeres en la prevención y solución de conflictos, al tiempo que promueve su intervención en procesos de toma de decisión y exhorta a los gobiernos a que tomen medidas para apoyar proyectos pacíficos de las mujeres locales. La resolución "[i]nsta a los Estados Miembros a velar por que aumente la representación de la mujer en todos los niveles de adopción de decisiones de las instituciones y mecanismos nacionales, regionales e internacionales para la prevención, la gestión y la solución de conflictos" (Consejo de Seguridad de Naciones Unidas, 2010: 2). Este tipo de instrumentos han sido implementados gracias al movimiento feminista global, que a su vez ha promovido los conceptos "agencia de las mujeres" y "empoderamiento en tiempos de guerra" (Zarkov, 2010).

A pesar de los avances, el nacionalismo y el feminismo son incompatibles en algunos países de Medio Oriente. Las mujeres siguen relegadas a la esfera privada y la opresión de que son víctimas se ha desplazado a un segundo plano.No obstante, en el caso de Palestina las mujeres han tenido un papel integral en el conflicto con Israel. La lucha de las palestinas ha evolucionado y se ha adaptado a los distintos momentos históricos. Esto ha ido desde manifestaciones en contra de asentamientos judíos a finales del siglo XIX, la creación de grupos armados femeninos en la década de los años treinta (Alsaafin, 2014), protestas contra el mandato británico en 1936, atentados militares realizados por revolucionarias como Leila Khaled en los años setenta, la fundación de diversas organizaciones no gubernamentales ${ }^{8}$ con objetivos nacionalistas y feministas en los años ochenta y noventa, la presencia política de mujeres en puestos de gobierno y, más recientemente, encuentros cara a cara con militares israelíes durante protestas pacíficas en aldeas palestinas, como fue el caso de Ahed Tamimi, encarcelada siendo menor de edad por abofetear a dos soldados israelíes en Nabi Saleh, Cisjordania.

\section{El feminismo egipcio a diez años de la "Primavera Árabe"}

El 25 de enero de 2011 estalló en Egipto una serie de revueltas populares como reacción al hartazgo de la sociedad ante la corrupción, los abusos del régimen autoritario, el desempleo, el aumento de precios a productos básicos, entre otras razones, que en términos generales tenían como objetivo la caída del régimen entonces encabezado por el expresidente Hosni Mubarak, quien llevaba treinta años en el poder y tenía planes de heredar la presidencia a su hijo Gamal. Luego de 18 días de protestas, Mubarak renunció, pero eso no evitó que las revueltascontinuaran. Éstas se extendieron hasta 2014, ya que en su segunda fase pretendían derrocar al miembro de la Hermandad Musulmana, Mohammad Morsi, quien había sido electo como presidente en los comicios llevados a cabo entre mayo y junio

8 Por ejemplo, el Centro de Asistencia Jurídica y Asesoría de la Mujer y la Sociedad de Mujeres Trabajadoras Palestinas para el Desarrollo. 
de 2012.

Las revueltas representaron un momento clave en la historia de movilización, no sólo de la población egipcia en general, sino de las mujeres en particular, ya que adquirieron distintas estrategias de contención ${ }^{9}$ política y, sobre todo, tomaron conciencia de su propia situación y capacidad de agencia. El papel que desempeñaron las egipcias se apoyó en distintas formas de contención: marchas, levantamientos, asambleas, desobediencia civil, declaraciones formales, publicaciones, organizaciones no gubernamentales, expresiones culturales y formas de confrontación modernas, como el activismo a través de las redes sociales de internet.

Se intensificó el uso de Facebook y Twitter, los cuales se convirtieron en una plataforma de expresión y difusión. Una de las figuras que utilizó este tipo de medios fue la joven Asma Mahfouz, quien mediante las redes sociales informó al mundo acerca de lo que sucedía en su país y animó a la sociedad egipcia a salir a manifestarse contra el gobierno corrupto el 25 de enero de 2011. En su videopublicado en YouTube días antes de que estallaran las protestas en la Plaza Tahrir de Egipto, destacaba la importancia de la reivindicación de los derechos y la lucha contra corrupción (Achmawi, 2011: 276).

El caso de Asma fue el más sonado en los medios de comunicación, pero también hubo otras mujeres que ganaron espacios en la web como Noha Atef, periodista egipcia que en 2006 comenzó a escribir en un blog sobre casos de tortura ocurridos durante el régimen de Mubaraky Nawara Negm, activista, periodista y bloguera que estuvo presente en los levantamientos de la Plaza Tahrir y fue informante de medios internacionales como Al Jazeera. Algunas de las formas de violencia que sufrieron las mujeres durante las revueltas fueron arrestos por parte de la policía y el ejército, quienes intentaron aterrorizar a la población mediante el uso excesivo de la fuerza, la cual iba desde golpes, amenazas, acoso sexual, hasta la realización de pruebas de virginidad. En este tema, se destaca el caso de Samira Ibrahim, detenida durante una protesta en marzo de 2011, quien denunció ante un tribunal egipcio haber sido golpeada, sometida a descargas eléctricas y a una prueba de virginidad por parte del ejército.

Durante las protestas, se crearon redes de solidaridad política transnacional, sobre todo a lo largo de algunos países de Medio Oriente. Las activistas egipcias compartían algunos objetivos con las tunecinas, las marroquíes y las sirias, entre ellos que se establecieran políticas para mejorar las condiciones económicas de cada país y, aunque las protestas res-

9 El término "contención" proviene de la teoría de movimientos sociales y se refiere al conjunto de medios, tácticas y estrategias que posee un grupo para hacer reclamos. Son, además, las formas en que las personas actúan juntas y persiguen intereses compartidos. Las personas se involucran en políticas contenciosas cuando surgen cambios en los patrones de oportunidades y limitaciones políticas y, luego, emplean estratégicamente un repertorio de acción, creando nuevas oportunidades para otros actores (Tarrow, 2011: 29) . 
pondieron a situaciones nacionales propias, desbordaron el marco nacional, dando una apariencia de contagio y de ola revolucionaria (Barreñada, 2012: 61).

El estudio del activismo feminista egipcio desde las Relaciones Internacionales es relevante debido a que involucra el análisis de las mujeres a modo de actores no clásicos en el sistema internacional y como agentes de cambio en la sociedad civil. En el marco de las revueltas populares árabes, las egipcias crearon redes transnacionales mediante organizaciones y movimientos, lo que las afianzó como actores de la escena internacional.

En sintonía con el estudio de las corrientes de género en las Relaciones Internacionales, pese a que el feminismo egipcio goza de una larga historia, el carácter feminista ${ }^{10}$ que se ha atribuido al activismo de las egipcias en distintos momentos de la historia ha sido muy discutido, ya que se ha llegado a considerar que el feminismo ha sido impuesto en las sociedades bajo el entendido de que sus discursos son universales, es decir, que todas las feministas tienen las mismas demandas y perspectivas y que no todas las activistas que defienden los derechos de las mujeres requieren el respaldo del feminismoproveniente del orden colonial y occidental.

Este argumento se inscribe precisamente en una perspectiva poscolonial, pues critica algunos feminismos hegemónicos de Occidente debido a distintas razones.Por mencionar algunas, consideran a las mujeres de Tercer Mundo (entre las cuales están las árabes y musulmanas) como sujetos monolíticos, homogéneos y oprimidos (Mohanty, 2008: 112), presuponen que la categoría "mujeres" es un grupo ya constituido con intereses idénticos sin importar la clase social, raza o etnia (Mohanty, 2008: 120), y definen y dividen a la sociedad en términos de un enfrentamiento entre las mujeres como víctimas y los hombres como sujetos que ejercen violencia (Mohanty, 2008: 124).

Y aunque las sociedades de mayoría musulmana, como la egipcia y la palestina, suelen ser distinguidas, casi de modo absoluto por los especialistas, sociólogos y antropólogos, como sociedades patriarcales (González, 2013: 495), dar por sentado que la dominación patriarcal es universal, sin mayores diferencias, conduce a una lectura eurocéntrica de las relaciones de género, es decir, bajo esta bandera de unidad se abre la posibilidad de transmitir los avances de la "modernidad" en el campo de los derechos a las mujeres no-blancas, indígenas y negras de los continentes que han sido colonizados. Se sustentaría, así, una posición de superioridad moral de las mujeres europeas o eurocentradas (Segato, 2010).

Debido a los argumentes anteriores, resultaimportante profundizar en la diversidad del feminismo, ya que, así como no se ha de simplificar al feminismo en Egipto, tampoco debe pensarse que todas las activistas en el exterior pretenden dar lecciones de feminismo a las egipcias; por el contrario, algunas tienen la intención de solidarizarse con otras luchas.

10 Como asevera Miriam Cooke (2001: VIII), el feminismo es mucho más que una ideología que impulsa movimientos políticos organizados. Es una actitud que resalta el papel del género en la comprensión de la organización de la sociedad. El feminismo implica la conciencia política e intelectual de la discriminación de género, el rechazo de conductas que fomentan dicha discriminación y la promoción de proyectos activistas para poner fin a la discriminación y abrir oportunidades para que las mujeres participen en la vida pública. 
Por ejemplo, la feminista estadounidense Angela Davis ha mostrado solidaridad con las feministas egipcias y de otras latitudes.

A este respecto, Laura Bier (2011: 40) argumenta que las nociones egipcias de los feminismos no han surgido únicamente a partir de preceptos occidentales, sino que han adoptado formas muy diversas. Prueba de esta situación son los casos de Huda Sharawi, considerada como la fundadora del feminismo egipcio, y Malak Hifni Nasif, conocida por su seudónimo, Bahithat al Badiya, cuyas opiniones sobre la posición de las mujeres se articularon dentro de una tradición modernista islámica, la cual sostiene que el islam es compatible con las bases de la civilización moderna, a saber, la razón, el progreso y la solidaridad. Por tanto, si vivir en el mundo moderno demandaba hacer cambios respecto a la organización de la sociedad, los musulmanes debían llevarlos a cabo sin abandonar sus principios (Hourani, 1991: 837).

Finalmente, las agendas de los organismos intergubernamentales también han influido en los feminismos palestino y egipcio, sobre todo mediante la ayuda financiera que brindan a organizaciones independientes de mujeres, la capacitación y profesionalización del personal de las dichas instituciones y el establecimiento de un marco jurídico internacional en torno a los derechos de las mujeres, como la Convención sobre la Eliminación de Todas las Formas de Discriminación contra la Mujer (CEDAW por sus siglas en inglés) de la ONU, la cual tiene el objetivo no solo de establecer conceptos universales relacionados con la desigualdad de género, sino también proponer una serie de medidas para erradicar las prácticas de maltrato hacia las mujeres y promover su representatividad en la esfera pública. Esta convención, adoptada en 1979, está integrada por treinta artículos y es, sin duda, la convención más relevante sobre asuntos de género y promoción de la igualdad de sexos. Fue ratificada por Egipto en 1981 y por Palestina en 2014.

Otro instrumento internacional relevante fue el Programa de Acción emanado de la Conferencia Internacional sobre Población y Desarrollo de la ONU celebrada en EI Cairo, en 1994. Los delegados y las delegadas que acudieron a la Conferencia coincidieron en que la igualdad y el empoderamiento de las mujeres es una prioridad mundial. El Plan de Acción incluye objetivos relacionados con la educación, especialmente para las niñas, y la reducción de la mortalidad materno-infantil. Aborda cuestiones relacionadas con la población, el medio ambiente, la familia, las migraciones, la prevención y el control del VIH/SIDA, las alianzas con organizaciones no gubernamentales, entre otros temas (ONU, 2020).

En el caso egipcio, a una década de la llamada "Primavera Árabe", las mujeres continúan viviendo bajo el control de un régimen autoritario y opresor, actualmente presidido por Abdel Fatah al Sisi, quien pese a que ha implementado políticas favorables paras las mujeres, como la prohibición y penalización de la mutilación genital femenina y el acoso sexual, ha hecho poco por impulsar la participación de las mujeres en el ámbito público. Ha 
promovido leyes que coartan la libertad de expresión y obstaculizan el trabajo de organizaciones no gubernamentales ${ }^{11}$, que desde décadas atrás han luchado por la defensa de los derechos de las mujeres. Esto, aunado a la continuidad de prácticas enraizadas en la sociedad egipcia, como el acoso sexual y la violencia doméstica, así como las desigualdades en el ámbito laboral ${ }^{12}$.

\section{Conclusiones}

A lo largo de este artículo se han señalado algunas razones por las cuales los enfoques de género son apropiados y relevantes para el análisis de temas de estudio en el campo de las Relaciones Internacionales. Entre estas razones se destaca su componente crítico y reflexivo, ya que a diferencia de teorías clásicas como el realismo político, los enfoques de género no solo pretenden describir los fenómenos y conflictos internacionales al suponer que los actores internacionales y las relaciones de poder son inmutables y permanentes, sino que pretenden contribuir a una transformación social en beneficio de relaciones de poder más equitativas, de la igualdad de género y del aumento de la participación política de los y las ciudadanas.

Pese a que en este artículo la reflexión sobre los objetos de estudio es breve, la intención es demostrar que, el análisis de los activismos feministas palestino y egipcio, en el marco de la ocupación israelí y de las revueltas populares árabes respectivamente, refleja la manera en que los conceptos y enfoques de género pueden ser aplicados para construir investigaciones innovadoras en la disciplina de las Relaciones Internacionales. Para ambos casos de estudio resultó útil emplear el enfoque feminista poscolonial o de Tercer Mundo, que, entre otras aportaciones, realiza una crítica a los estereotipos que existen acerca de la supuesta sumisión y pasividad de las mujeres árabes y musulmanas.

Para cerrar, dado el predominio del machismo y la violencia sistémica contra las mujeres en todo el mundo, es necesario visibilizar las distintas formas de activismos feministas y las redes transnacionales de mujeres, las cuales son materia prima para diversificar los temas de estudio en las Relaciones Internacionales, desviar la atención del análisis de las relaciones interestatales y dirigirla hacia un estudio de las mujeres como actores de alcance transnacional, con el fin de situar a las construcciones de género y a las mujeres en el centro de las investigaciones.

11 Por ejemplo, la Ley número 70 de 2017 que regula el registro y el funcionamiento de todas las entidades de la sociedad civil en Egipto. La ley implica distintos obstáculos para las organizaciones: señala que las asociaciones deben limitarse a llevar a cabo actividades en los campos del desarrollo y el bienestar social y que el gobierno egipcio tiene la autoridad para inspeccionar las sedes de las asociaciones en cualquier momento e interferir en sus asuntos internos (International Center for Not-for-Profit Law, 2019)

12 Datos de la Organización Internacional del Trabajo (OIT, 2019) revelan que, en 2017, la tasa de participación femenina (mayor de 15 años de edad) en la fuerza laboral era del $22 \%$, esto frente a un $66.9 \%$ en los hombres. 


\section{Fuentes de consulta}

Abu Ghaida, D. (1993). Secular Feminism vs. Islamist Feminism in Palestine.The Arab Studies Journal, 1 (2), 18-20.

Achmawi, R. (2011). El papel de las mujeres en la Primavera Árabe. Quaderns de la Mediterànea, 16, 273-281.

Alsaafin, L. (17 de abril de 2014). The role of palestinian women in resistance.Open Democracy. https://www.opendemocracy.net/en/north-africa-west-asia/role-ofpalestinian-women-in-resistance/

Álvarez-Ossorio, I. (3 de marzo de 2020).Trump y el expolio de Palestina. El País. https://elpais.com/elpais/2020/02/03/opinion/1580755580_154417.html.

Barreñada, I. (2012). El continuum contestatario en los países árabes. Movimientos sociales, sociedad civil y ciudadanía. Regions \& Cohesion, 2 (3), 45-66.

Bier, L. (2011). Revolutionary Womanhood: Feminisms, Modernity, and the State in Nasser's Egypt. Stanford University Press.

Butler, J. (24-27 de junio de 2014). Rethinking vulnerability and resistance [Conferencia Plenaria]. XV Simposio de la Asociación Internacional de Filósofas. Universidad de Alcalá. https://www.youtube.com/watch?v=hEjQHv0R6rQ.

Castells, M. (2008). The New Public Sphere: Global Civil Society, Communication Networks, and Global Governance. ANNALS. American Academy of Political and Social Science, 616, 78-93.

Consejo de Seguridad de Naciones Unidas. (2010). Resolución 1325. https://www.un.org/womenwatch/ods/S-RES-1325 (2000) -S.pdf.

Cooke, M. (2001).Women Claim Islam. Creating Islamic Feminism through Literature. Routledge.

D’Amico, F. (1994). Pluralist and critical perspectives. En P. Beckman y F. D’Amico (Eds.), Women, Gender, and World Politics: Perspectives, Policies, and Prospects. Bergin \& Garvey.

Enloe, C. (2014). Bananas, Beaches and Bases: Making Feminist Sense of International Politics. University of California Press.

GonzálezA. (2013). Los conceptos de patriarcado y androcentrismo en el estudio sociológico y antropológico de las sociedades de mayoría musulmana. Papers. Revista de Sociología, 98 (3), 489-504.

Harding, S. (1986). The science question in feminism, Estados. Open University Press.

Hourani, A. (1991).A history of the Arab peoples.Faber and Faber.

International Center for Not-for-Profit Law. (2019).Civic Freedom Monitor, Egypt. https://www.icnl.org/resources/civic-freedom-monitor/egypt.

Keohane R. O. y Nye, J. S. (1971). Transnational relations and world politics: an introduction. 
International Organization, 25 (3), 329-349.

Kuttab, E. (2009). The Palestinian Women's Movement: From Resistance and Liberation to Accommodation and Globalization.Vents d'Est, vents d'Ouest. Mouvements de femmes et féminismes anticoloniaux, 101-116.

Mohanty, C. (2008). Bajo los Ojos de Occidente: Feminismo Académico y Discursos Coloniales. En L. Suárez y R. Hernández (Eds.), Descolonizando el Feminismo: Teorías y Prácticas desde los Márgenes. Cátedra.

Morgenthau, H. (1997). Politics Among Nations. Peking University Press.

Ochoa, L. et al. (2016).El Surgimiento y el Desarrollo de la Disciplina de las Relaciones Internacionales. En J. Schiavon et al. (Eds.), Teorías de las Relaciones Internacionales en el siglo XXI. Interpretaciones críticas desde México (pp. 29-45). CIDE.

OIT Organización Internacional del Trabajo. (3 de abril de 2019).Key Indicators of the labour market. https://www.ilo.org/ilostat/faces/wcnav_defaultSelection?adf.ctrlstate=ursxsqhzo_4\&_afrLoop=3199795951309325\&_afrWindowMode=0\&_afrWindowld=ursxsqhzo_75\#!el.

ONU. (2020). Conferencia Internacional sobre Población y DesarroIlo.https://www.un.org/es/events/pastevents/icpd_1994/.

Runyan, A. y Peterson, V. S. (2014).Global Gender Issues in the New Millennium. Westview Press.

Salomón, M. (2002). La teoría de las relaciones internacionales en los albores del siglo XXI: diálogo, disidencia, aproximaciones. Revista Electrónica de Estudios Internacionales, 4, 1-59.

Segato, R. (2010). Género y colonialidad: en busca de claves de lectura y de un vocabulario estratégico descolonial. En A. Quijano y J. Mejía (Eds.), La Cuestión Descolonial. Universidad Ricardo Palma. Cátedra América Latina y la Colonialidad del Poder.

Shaw, M. (1994). Civil society and global politics: beyond a social movements approach.Millennium: Journal of International Studies, 23 (3), 647-667.

Smith, J. (10-12 de mayo de 2002). Globalization and Transnational Social Movements Organizations [Conferencia Plenaria]. Conference on Struggles and Settlements in Organizations and Movements. Michigan. http://citeseerx.ist.psu.edu/viewdoc/download?rep=rep1\&type=pdf\&doi=10.1.1.194.6320.

Tarrow, S. (2011).Power in Movement: Social Movements and Contentious Politics. University Press.

Tickner, A. (2008). Gender in World Politics. En J. Baylis et al., The Globalization of World Politics. Oxford University Press.

Tickner, A. (1988). Hans Morgenthau's principles of political realism: a feminist reformulation. Millennium: Journal of International Studies, 17 (3), 429-440. 
True, J. (2008). The ethics of feminism. En C. Reus-Smit y D. Snidal (Eds), The Oxford Handbook of International Relations. Oxford Handbooks.

Villarroel, Y. (2007). Los aportes de las teorías feministas a la comprensión de las relaciones internacionales. Politeia, 39 (30), 65-86.

Whitworth, S. (1994). Feminist theories: from women to gender and world politics. En P. Beckman y F. D'Amico (Eds.), Women, Gender, and World Politics: Perspectives, Policies, and Prospects. Bergin \& Garvey.

Zarkov, D. (2010). Feminist Conceptualization of War; Geographies of Thinking. Review of Women's Studies, 6, 12-24. 